\title{
Applications of multiphoton polarization and generalized polarization microscopy in elucidating transdermal delivery pathways
}

\author{
Yen Sun ${ }^{\mathrm{a}}$, Jiunn-Wen Su ${ }^{\mathrm{a}}$, Wen Lo ${ }^{\mathrm{a}}$, Sung-Jan Lin ${ }^{\mathrm{b}}$, Shiou-Hwa Jee ${ }^{\mathrm{b}, \mathrm{c}}$, Chen-Yuan Dong ${ }^{\mathrm{a}}$ \\ ${ }^{a}$ Department of Physics, National Taiwan University, Taipei 106, Taiwan \\ ${ }^{b}$ Department of Dermatology, National Taiwan University Hospital, Taipei 100, Taiwan \\ ${ }^{\mathrm{c}}$ Department of Dermatology, College of Medicine, National Taiwan University, Taipei 100, Taiwan
}

\begin{abstract}
Physical and chemical enhancement techniques have proven to be useful in promoting transdermal delivery of therapeutic agents. However, it has been difficult to follow the changes to the skin during these processes microscopically. In this work, we use multiphoton polarization (P) and generalized polarization (GP) imaging of the membrane probe Laurdan to monitor changes to the stratum corneum under conditions of chemically enhanced delivery by oleic acid. We show that the combination of P and GP Laurdan imaging helps to reveal both the physical and chemical changes to the stratum corneum under enhanced delivery. Our work can help to develop more efficient transdermal delivery strategies.
\end{abstract}

Keywords: multiphoton microscopy, polarization, generalized polarization, skin, transdermal delivery

*To whom correspondence should be addressed: cydong@phys.ntu.edu.tw 


\section{Introduction}

\subsection{Principles of multiphoton microscopy}

In the decade since the introduction of multphoton fluorescence microscopy, research in the life sciences have benefited greatly from the continual development of this technique. A number of significant advantages are associated with this imaging modality. First, the high photon flux necessary for generating the non-linear fluorescence excitation limits sample excitation to the focal volume. Imaging using the point-like excitation volume results in images with excellent axial depth discrimination without the use of confocal apertures. In addition, the confined sample excitation also limits sample photodamage. Furthermore, the near-infrared wavelength photons used for sample excitation are scattered and absorbed less than the ultraviolet or visible photons used in typical one-photon microscopy. As a result, multiphoton imaging allows greater sample imaging depths to be achieved without histological procedures. Finally, the wide spectral separation between the excitation and sample luminescence allows easy separation of the signal photons from that of the excitation source and the complete emission spectrum can be easily obtained. ${ }^{1,2}$ These advantages have been utilized by researchers in addressing a number of significant research areas in the life sciences such as developmental biology, neurobiology, deep tissue imaging, and transdermal delivery. ${ }^{3-7}$

\subsection{Polarization and generalized polarization}

In the field of transdermal delivery, it may be important to monitor the physical and chemical changes to the skin under the action of different enhancement procedures. For fluorescence microscopy, two parameters may be used for the characterization of skin changes. First, polarization P defined as

$$
P=\frac{I_{I I}-I_{\perp}}{I_{I I}+I_{\perp}}
$$

is one such parameter. In the above definition, $I_{I I}$ and $I_{\perp}$ are the fluorescence intensity parallel and perpendicular to the incident excitation polarization. ${ }^{8}$ As Fig. 1 shows, if the lipid packing at the skin surface is disrupted for the facilitation of the delivery process, then it is conceivable that the lipid packing may be disturbed to facilitate this process. Therefore, under polarized excitation, one would expect the fluorescence polarization value to decrease, and polarization microscopy may be an useful technique to monitor such physical changes.

Another technique which may be used to monitor chemical changes to the lipid environment is generalized polarization (GP) microscopy. The definition of GP is given as

$$
G P=\frac{I_{440}-I_{490}}{I_{440}+I_{490}}
$$


where $I_{440}$ and $I_{490}$ are the fluorescence emission intensity of the membrane probe Laurdan at 440 and $490 \mathrm{~nm}$, respectively. It has been shown that the unique spectral properties of Laurdan may be used to monitor the chemical environments of the molecules. Specifically, Laurdan has been shown to undergo red shift in a more polar environment. Therefore, one would expect the GP value associated with Laurdan to decreases when the molecules become exposed to polar solvent molecules. ${ }^{9}$ Therefore, this work is intended to apply both the polarization and GP imaging of Laurdan molecules in the skin to understand the changes to skin surface when the chemical enhancer oleic acid is applied.

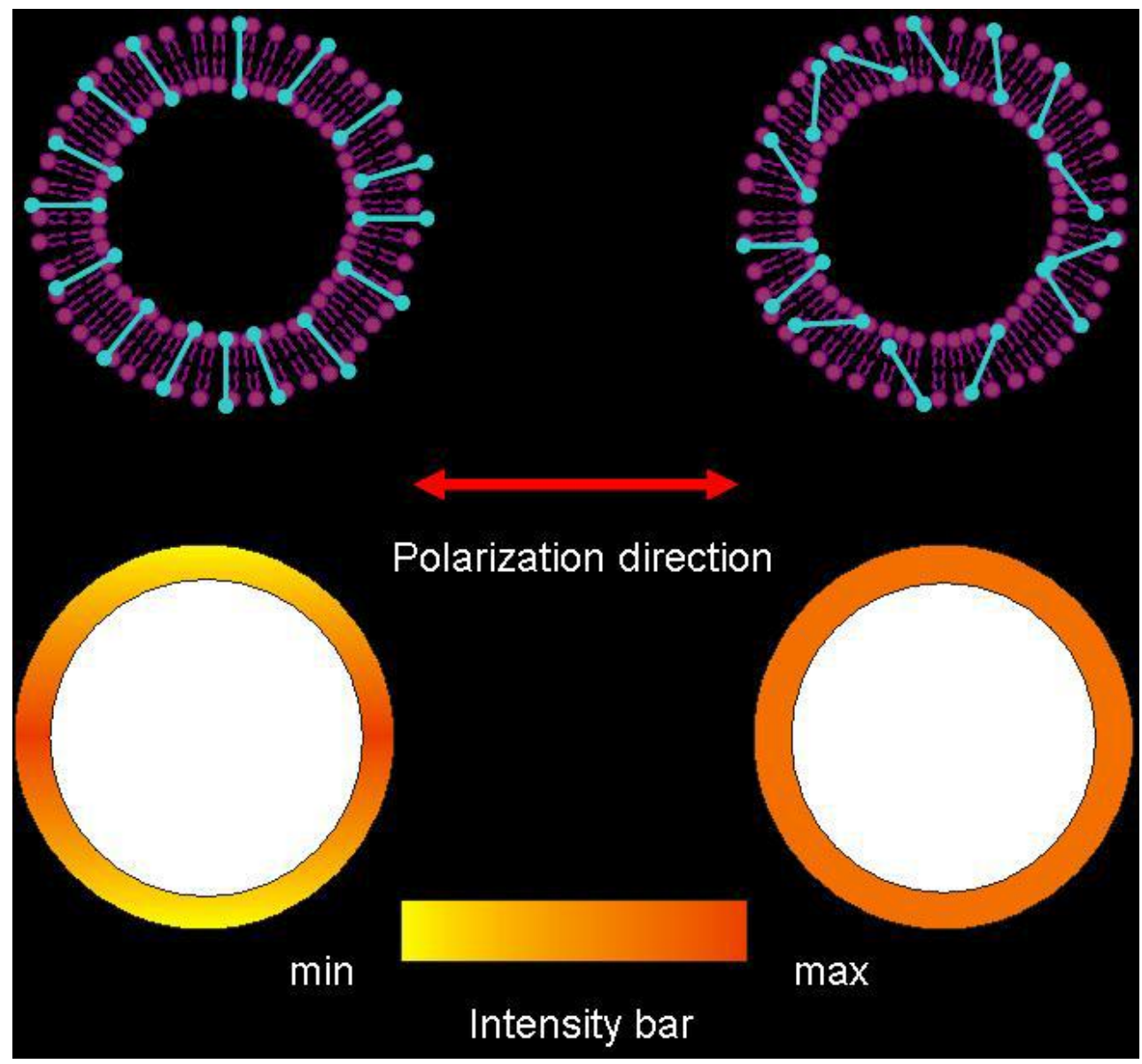

Fig. 1 Polarization effects in lipid structures

\section{Materials and Methods}

\subsection{A multiphoton microscope}

The multiphoton microscope used has been described. ${ }^{10}$ In short, the $780 \mathrm{~nm}$ output of a diode-pumped (Millennia X, Spectra Physics, Mountain View, CA), femtosecond titanium-sapphire laser system (Tsunami, Spectra Physics) was used as the excitation source. The laser beam is scanned by an x-y scanning system (Model 6220, Cambridge Technology, Cambridge, MA) prior to entering a modified commercial upright microscope system (E800, Nikon, Japan). The laser beam is beam expanded by a pair of beam expanding lenses at the entrance port of the microscope and is then reflected 
by a dichroic mirror (Chroma Technology, Brattleboro, VT) into the focusing objective. The Laurdan fluorescence was collected in the epi-illuminated fashion. The fluorescence was collected by the same focusing objective, passed through the dichroic and additional filters prior to being detected by the PMT (R7400P, Hamamatsu, Japan) and the signal photons were processed in the single-photon counting mode using a home-built discriminator. To implement multiphoton GP imaging two band pass filters centered at 440 nm (HQ 440-40m, Chroma Technology) and 490 nm (HQ 490-40m, Chroma Technology) were used to acquired spectrally images from Laurdan treated skin specimens. To obtain the polarization resolved images, a sheet polarizer was placed in front of the PMT and Laurdan fluorescence images were acquired at crossed orientations. To ensure equal excitation of the Laurdan molecules at different orientations within the lipids layers, the incident laser was made to be circularly polarized.

\subsection{Sample preparation}

Two types of the Laurdan labeled skin specimens were prepared. Excised human skin were obtained and used in both cases. In the first specimen in the absence of oleic acid, the skin was treated in a solution composed of $10 \mu 1$ of 2 mM Laurdan (6-dodecanoyl- 2-dimethylaminonaphthalene, D-250, Molecular Probes, Eugene, OR) stock (in DMSO) and $1 \mathrm{~mL}$ of PBS buffer. For the oleic acid labeled specimen, the formula of the labeling solution has been changed to be composed of $10 \mu \mathrm{l}$ of the Laurdan stock, $53 \mu \mathrm{l}$ of oleic acid, and $1 \mathrm{ml}$ of PBS. Both specimens were labeled for 10.5 hours. After the incubation period, the skin samples were rinsed in PBS and wiped prior to being mounted for multiphoton imaging.

\section{Results and Discussion}

The multipoton images acquired and processed are shown in Fig. 2. After the raw images were acquired, both the polarization (P) and generalized polarization (GP) images were computed for the cases with and without the treatment of oleic acid. In examining the polarization images (Fig. 2a and 2c), one sees that without the oleic acid treatment, polarization images tend to be more polar than the results with oleic acid treatment. To quantify this observation, the histograms of the polarization values are plotted in Fig. 3. Furthermore, six regions of the polarization values (three vertical and three horizontal) were analyzed. These results are also shown in Fig. 3. The average polarization values for Fig. $2 \mathrm{a}$ and $2 \mathrm{c}$ are respectively $-0.01 \pm 0.18$ and $-0.02 \pm 0.16$. Although the average polarization values did not show significant differences with or without oleic acid treatment, analysis of the six differently oriented regions show a different story. The results in Fig. 3e show that without oleic acid treatment, the average polarization values for the lipid layers oriented at horizontal and vertical directions are respectively $-0.09 \pm 0.08$ and $0.09 \pm 0.11$. In our analysis of the polarization results, the $I_{I I}$ and $I_{\perp}$ are defined as the fluorescence intensity horizontal and vertical to the image orientation. Therefore, the polarization values we obtain are consistent with the fact that in the absence of oleic acid, Laurdan molecules are oriented normal to the lipid layers. In comparison, Fig. $3 \mathrm{f}$ shows that with oleic acid treatment, the polarization values are $-0.01 \pm 0.08$ and $-0.01 \pm 0.08$ regardless of the lipid layer orientations. Therefore, our results show that as a result of the fact that polarization values of differently oriented lipid layers were averaged, the global polarization histograms do not reveal significant difference between differently oriented lipid layers. However, detailed 
analysis of the skin lipid layers oriented at different orientations show that oleic acid has a tendency to randomize the orientation of lipid molecules within the skin.

The GP images were analyzed in a similar fashion. As in the case of polarization images, the GP values of the six regions were investigated and the results are shown in Fig. 3e and $3 \mathrm{f}$. Our results show that regardless of the lipid orientations, the addition of oleic acid has a tendency to lower the GP value. This observation supports the conclusion that the not only does oleic acid perturbs the orientation of lipid molecules within the skin, the perturbation also allows a more polar environment to exist within the skin layers. To investigate this phenomenon in greater details, consider the GP images and histograms with and without oleic acid treatment. The histograms in Figs. $2 \mathrm{~b}$ and $2 \mathrm{~d}$ show a shift towards lower values of GP upon the addition of oleic acid. Across the entire image, the average GP values decreased from $0.35 \pm 0.14$ to $-0.06 \pm 0.15$ once oleic acid is added. Therefore, our multiphoton GP images support the fact that oleic acid functions by disturbing the lipid molecular packing on the skin and allows the penetration of water molecules into the lipid layers.

Finally, our results show that within the skin, different regions of different polarity exist. Two regions in the GP images were selected and analyzed. Specifically, in Fig. 2b, in the case without oleic acid treatment, the GP values for regions b1 and b2 are $0.37 \pm 0.15$ and $0.27 \pm 0.14$ respectively. Similar analysis in two regions in the case where oleic acid was added shows that the GP values are $-0.08 \pm 0.13$ and $-0.11 \pm 0.11$ for regions $\mathrm{d} 1$ and $\mathrm{d} 2$, respectively.

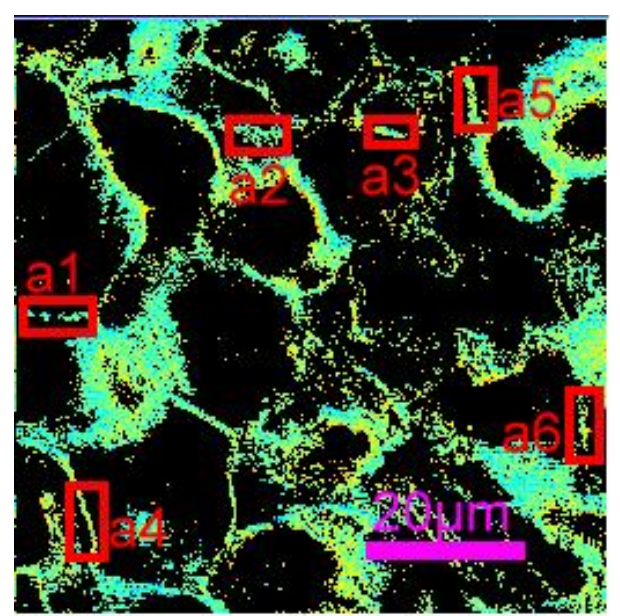

a) P: without OA

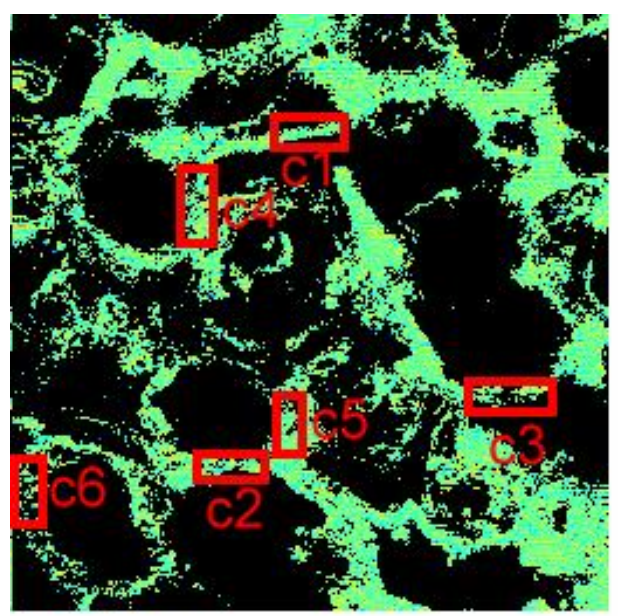

c) P: with OA 


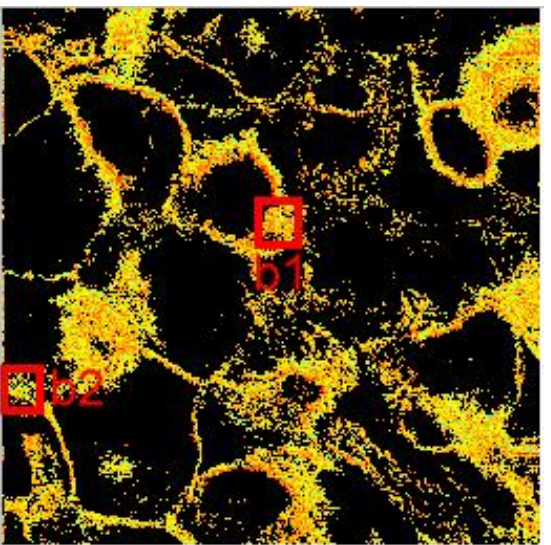

b) GP: without OA

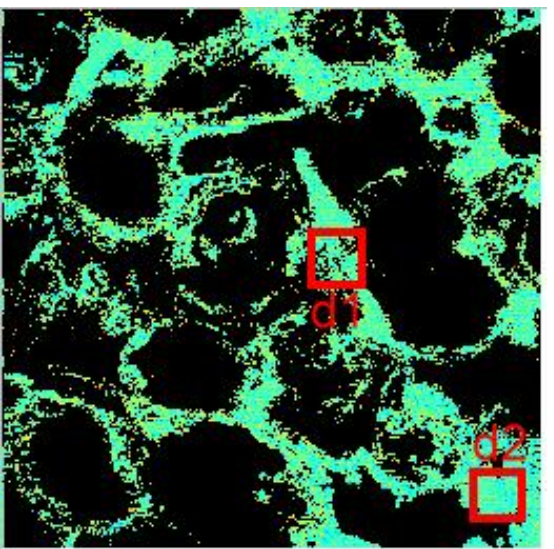

d) GP: with OA

$-1$

Fig. 2 Laurdan skin P images a) without and c) with the addition of oleic acid. Laurdan GP images b) without and d) with the addition of oleic acid $\left(I_{\max }=1, I_{\min }=-1\right)$. In b) and d), two areas are chosen for GP analysis. In b), the GP's for areas b1 and b2 are respectively $0.37 \pm 0.15,0.27 \pm 0.14$. In d), the GP values for regions $\mathrm{d} 1$ and $\mathrm{d} 2$ are $-0.08 \pm 0.13$ and $-0.11 \pm 0.11$, respectively.

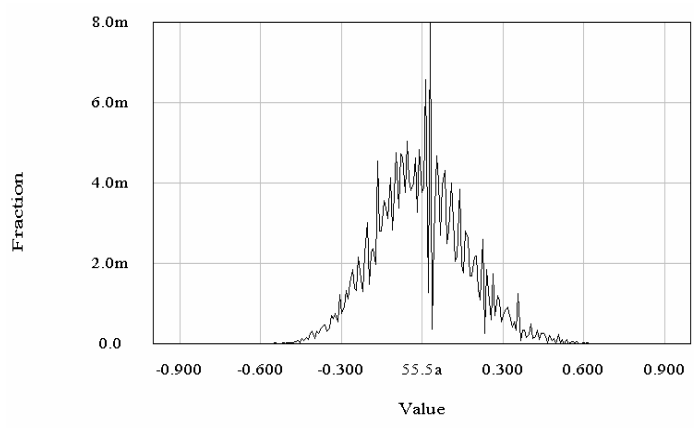

a) P: without OA

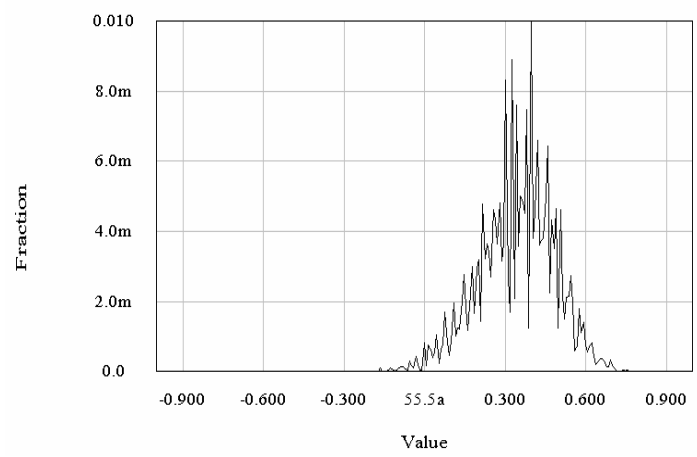

b) GP: without $\mathrm{OA}$

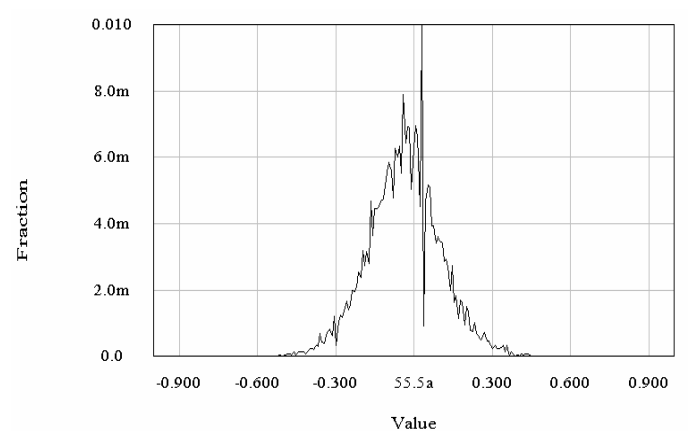

c) P: with $\mathrm{OA}$

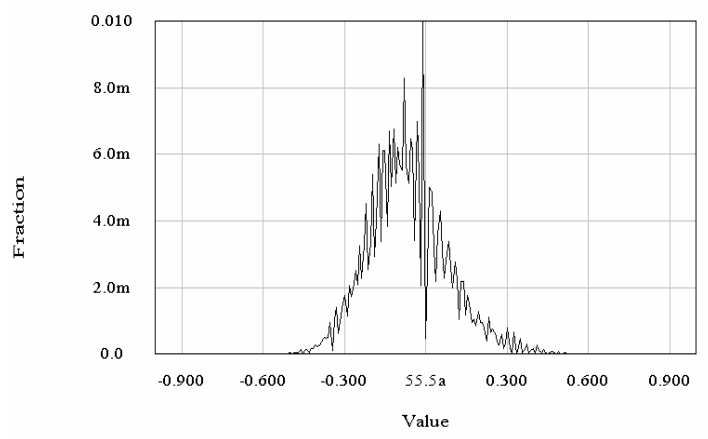

d) GP: with OA 


\begin{tabular}{|c|c|c|c|c|c|}
\hline \multicolumn{6}{|c|}{ Without Oleic Acid } \\
\hline membrane orientation & area & Pavelag & GPareage & P total areelage & GP total areagege \\
\hline \multirow{3}{*}{$\longleftrightarrow$} & 1 & 0.11 & 0.33 & \multirow{3}{*}{0.0800 .08} & \multirow{3}{*}{$0.34+0.08$} \\
\hline & 2 & 0.08 & 0,35 & & \\
\hline & 3 & 0.03 & 0.35 & & \\
\hline \multirow[b]{3}{*}{ 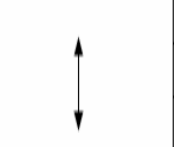 } & 4 & 0.13 & 0.37 & \multirow{3}{*}{0.0950 .11} & \multirow{3}{*}{$0.35+0.08$} \\
\hline & 5 & 0.07 & 0,32 & & \\
\hline & 6 & 0.1 & 0.36 & & \\
\hline
\end{tabular}

e) P: without $\mathrm{OA}$ at chosen regions

\begin{tabular}{|c|c|c|c|c|c|}
\hline \multicolumn{6}{|c|}{ With Oleic Acid } \\
\hline membrane orientation & area & Paverage & GP arelage & Ptotal areage & GP total arealage \\
\hline \multirow{3}{*}{$\longleftrightarrow$} & 1 & 0.02 & 0.03 & \multirow{3}{*}{$0.0100,08$} & \multirow{3}{*}{$0.04+0.09$} \\
\hline & 2 & 0.01 & 0.03 & & \\
\hline & 3 & 0.01 & 0.07 & & \\
\hline \multirow[b]{3}{*}{1} & 4 & 0.02 & .0 .07 & \multirow{3}{*}{$\sqrt{0.01+0.08}$} & \multirow{3}{*}{$0.0550,0.08$} \\
\hline & 5 & 0.01 & 0.07 & & \\
\hline & 6 & 0.01 & 0.02 & & \\
\hline
\end{tabular}

f) $\mathrm{P}$ : with $\mathrm{OA}$ at chosen regions

Fig. 3 Laurdan polarization histograms in a) the absence and c) the presence of oleic acid. Laurdan GP histograms b) without and d) with oleic acid. OA = oleic acid. e) and f) are the $\mathrm{P}$ and GP results without and with OA, respectively, at selected regions.

\section{Conclusions}

In conclusion, we have demonstrated the usefulness of multiphoton polarization and generalized polarization (GP) imaging in understanding the changes to skin in the presence of chemical enhancer oleic acid. Multiphoton polarization imaging is useful in revealing the changes in lipid molecule alignments while GP microscopy reveals the associated changes in the chemical environment. A combination of multiphoton polarization and GP imaging is of great value in understanding biological transport phenomena.

\section{ACKNOWLEDGMENT}

We acknowledge the support of NSC NSC 92-2112-M-002-018 and NSC 92-3112-B-002-048 (National Science Council, Taiwan) for this project.

\section{REFERENCES}

1. W. Denk, J. H. Strickler, and W. W. Webb, "2-Photon Laser Scanning Fluorescence Microscopy," Science 248(4951), 73 (1990).

2. P. T. C. So, C. Y. Dong, B. R. Masters, and K. M. Berland, "Two-photon excitation fluorescence microscopy," Annual Review of Biomedical Engineering 2, 399 (2000).

3. J. M. Squirrell, D. L. Wokosin, J. G. White, and B. D. Bavister, "Long-term two-photon fluorescence imaging of mammalian embryos without compromising viability," Nat. Biotech. 17, pp. 763-767, 1999.

4. K. Svoboda, K, W. Denk, D. Kleinfeld, and D. W. Tank, "In vivo dentritic calcium dynamics in neocortical pyramidal neurons," Nature. 385, pp. 161-165, 1997.

5. P. T. C. So, H. Kim, and I. E. Kochevar, "Two-photon deep tissue ex vivo imaging of mouse dermal and subcutaneous structures," Opt. Exp. 3, 339-350, 1998. 
6. B. Yu, C. Y. Dong, P. T. C. So, D. Blankschtein, and R. Langer, "In vitro visualization and quantification of oleic acid induced changes in transdermal transport using two-photon fluorescence microscopy," Journal of Investigative Dermatology 117(1), 16 (2001).

7. B. Yu, K. H. Kim, P. T. C. So, D. Blankschtein, and R. Langer, "Topographic heterogeneity in transdermal transport revealed by high-speed two-photon microscopy: Determination of representative skin sample sizes," Journal of Investigative Dermatology 118(6), 1085 (2002).

8. J. R. Lakowicz, Principles of Fluorescence Spectroscopy, (Plenum, New York, 1999).

9. L. A. Bagatolli and E. Gratton, "Two photon fluorescence microscopy of coexisting lipid domains in giant unilamellar vesicles of binary phospholipid mixtures," Biophysical Journal 78(1), 290 (2000).

10. Y. Sun, W. Lo, W., S. J. Lin, S. H. Jee, and C. Y. Dong, "Multiphoton polarization and generalized polarization (GP) microscopy reveals oleic acid induced structural changes in intercellular lipid layers of the skin," Optics Letters, 29(17), 2013 (2004). 